\title{
Applications of Graphene in Medicine and Electronics: A Review
}

\author{
*Yagya Gera \\ M.Sc. Physics \\ Corresponding Author: Yagya Gera
}

\begin{abstract}
Carbon is unique in its own ways, the most special attribute of carbon is that it can make rings and long chains; even life on the earth is possible due to this property of carbon. Graphene is the first twodimensional material and it has many outstanding properties which have resulted in many exciting applications. Graphene is claimed to be so versatile that it will be able to replace present day materials. It is thought to be the perfect two-dimensional carbon crystal as it is the unique blend of properties both mechanical and electronics. Graphene is the thinnest, highly elastic, ambipolar, chemically inert, hydrophobic, possesses record electron conductivity, high value of mobility, high thermal conductivity, high opacity, low resistivity and shows fractional quantum Hall effect and ballistic conduction and many other interesting properties that are yet be explored. Recently it has been found that the magnetic field of graphene can be switched on and off. In this paper, I have done a comparative study of properties of graphene and tried to write a review on application of graphene in two fields which affect us most, that are, Medicine and Electronics.
\end{abstract}

Keywords: Graphene, properties ,tissue engineering, bio-micro robotics, drug delivery, bio-imaging, transistors, spin transport device, hall effect sensors, transparent conducting electrodes, conductive ink, quantum dots, photodetector, LED, optical limiters, touch screen, terahertz devices.

\section{Introduction}

Graphene is a two-dimensional atomic-thick hexagonal lattice in which one atom form each vertex. It is the basic building element of the other allotropes of carbon such as graphite, charcoal, carbon nanotubes and fullerenes. It can be considered as an indefinitely large layer of aromatic rings. Atomic thick, free-standing standing graphene from bulk graphite was extracted by Andre Geim and Konstantin Novoselov, Manchester University [1], U.K. in 2004 for which they received Nobel prize in 2010.Graphene is a member of twodimensional material family. With Graphyne, Borophene, Germanene, Silicene, Si2BN, Stanene, Phosphorene, Molybdenite, two-dimensional metal layers and others in this group, graphene was first to be discovered [2].

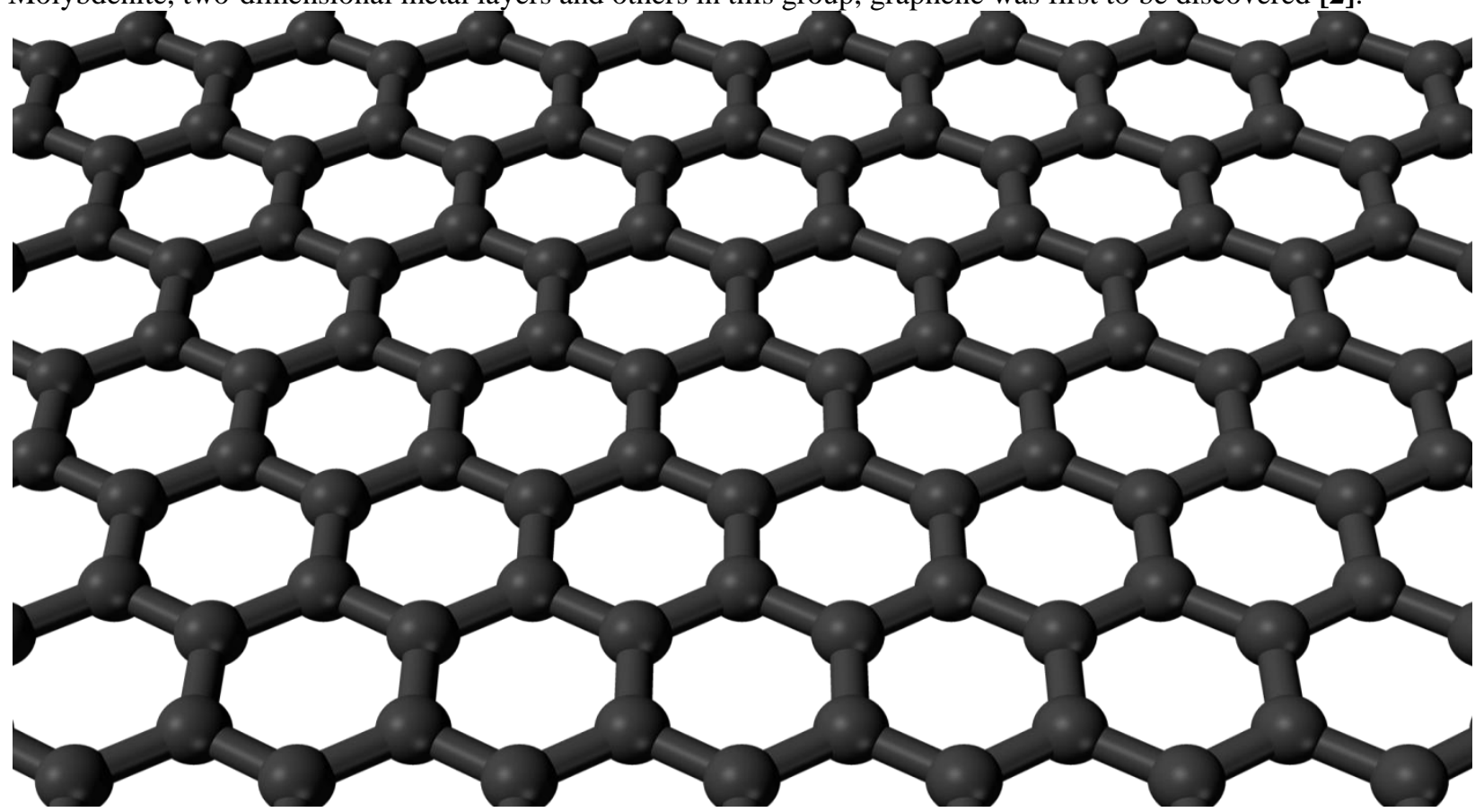

Fig 1: The unique Hexagonal Lattice Structure of carbon atoms forming Graphene. 
Its name was given by Hanns-Peter Boehm in 1962 [3], Graphite + -ene = Graphene. Even before 2010, when Andre Geim and Konstantin Novoselov received nobel prize for their work on graphene, in October 2009, IBM researchers used graphene to develop ultra-fast photo-detectors [4]. In November 2009 two companies Samsung and Fujitsu started their work on graphene. Samsung started doing research on graphene where Fujitsu started producing graphene transistor at low temperature. In December 2009 Angstrom materials was awarded one and half billion US dollars for production of nano-graphene platelets [4]. In February 2010 IBM manufactured a $100 \mathrm{GHz}$ transistor on 2 " wafer which operates at room temperature. It was found to be double times faster than silicon transistor for the same gate length [4].IBM later developed a 155GHz transistor and $10 \mathrm{GHz}$ graphene-based nanowire. UCLA in September 2010 developed a $300 \mathrm{GHz}$ transistor using a nanowire. Samsung which started with its research in graphene became the company that possesses the highest number of graphene patents in the world. Recently Samsung has announced a foldable phone. With so many potential applications governments of different countries started investing in graphene research. The European Union granted 1 billion euro for graphene research. Sweden, on the other hand, invested 6 million US Dollars in graphene research. South Korea on the other invested 40 million US Dollars in the commercialization of Graphene [4]. Recently, commercial growth and development of graphene has become multifold. UCLA starting selling laser scribed graphene supercapacitor [4]. IBM developed a graphene insulator super-lattice which can achieve a Terahertz frequency and can be used as Terahertz filter and polarizer. China developed 15"transparent graphene film.Graphene has been the center of attraction from many recent years due to its outstanding properties. With lots of noteworthy properties, the market of graphene is expected to rise 278.47 USD by 2020 [4].If development were like this, it will affect each and every life on earth. The excellence of the graphene lies in packing of carbon atoms in a flat honeycomb structure. The structure of graphene has three carbon atoms bonded to each carbon atom. Thus fourth valance electron remains free and a group of electrons is formed.

\subsection{Properties of Graphene}

1.1.1 Size

It is thinnest material known, just one atom thick! With thickness of about 0.4-1.7nm [5], it is about one million times thinner than human hair .Its thinness allows it to be extremely flexible and at the same time conduct heat and electricity. It is hardest material known to mankind, even harder than diamond. Tensile strength of Graphene is 130GPa [6] whereas tensile strength of diamond so far observed is 60GPa which theoretically may rise up to $225 \mathrm{GPa}$.Depending upon crystal orientation. Graphene is extremely light. Its weight per square meter is $0.77 \mathrm{mg}$ which is 1000 times lighter than paper. 1 gram of graphene is sufficient to cover $5500 \mathrm{~m} 2$ area which is roughly area of a football field [7].

\subsubsection{Elasticity}

Graphene is highly elastic. Sheets of graphene with thickness ranging from 2-8nm was observed to have spring constant of 1 to $5 \mathrm{Nm}^{-1}$. and Young's modulus of $0.5 \mathrm{TPa}$ and bulk modulus of 1TPa. [8] .Diamond on the other hand has bulk modulus of 443GPa and Young's modulus of $1220 \mathrm{GPa}$.

\subsubsection{Conductivity}

Graphene shows record electron conductivity. With zero band gap, the bands of graphene meet at Dirac points. Graphene is a two-dimensional layer of aromatic rings with its hydrogen atoms ripped off. Each atom is connected to three other atoms in 2 dimensional layer. Thus one electron is available in third dimension. These free electrons are located in pi orbital and so are called pi electrons. They are located up and below the graphene sheets. They are highly mobile and are able to travel up to micrometers without scattering at room temperature. These orbital overlap and strengthen the carbon to carbon bonds .Bonding and anti-bonding of these pi orbital dictate the electronic properties of graphene.Electronic mobility of these electron is $200,000 \mathrm{~cm}^{2}$ $\mathrm{V}^{-1} \mathrm{~s}^{-1}$ at electron densities of $\sim 2 \times 10^{11} \mathrm{~cm}^{-2}$ [9]. This high value of mobility of electrons is comparable to mobility of photons. This is due to reason that electrons in graphene do not have mass at Dirac points. .Graphene is around $10^{6}$ times more conductive electrically measured at room temperature than copper. [10].

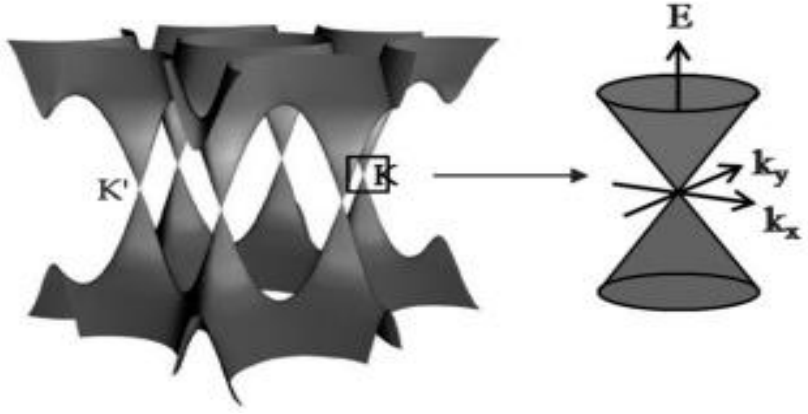

Fig. Electronic Band Structure of Graphene. 


\subsubsection{Thermal Conductivity}

Graphene has extremely high thermal conductivity. Thermal conductivity of graphene at room temperature is about 2000 to $4000 \mathrm{Wm}^{-1} \mathrm{k}^{-1}$ [11]. And for diamond it lies between $2200-3300 \mathrm{Wm}^{-1} \mathrm{~K}^{-1}$. [11]

OPACITY: One atomic monolayer of graphene has high opacity but is visible to naked eye. It absorbs $2.3 \%$ of red light and $2.6 \%$ of green light. [12]

RESISTIVITY : Graphene possess Lowest Resistivity at room temperature. The resistivity of the graphene sheet is found to be $10^{6} \mathrm{ohm}-\mathrm{cm}$, which is very less than that of silver. [13]

\subsubsection{BALLISTIC CONDUCTION}

Ballistic conduction is motion of electron due to scattering in a medium where electrical resistivity almost zero. For this to happen the mean free path of electron that is the path travelled by the electron before collision is much greater than the size of the medium. Ballistic conduction is unrestricted flow of charge carriers. It is different from the superconductivity due to the reason that electrons stop flowing once the driving force is turned off but in superconductivity the charge carriers keep on flowing even if the external driving force is withdrawn. Ballistic conduction is possible in one-dimensional materials due to small size of such materials. Graphene ribbons show exceptional ballistic transport properties.

\subsubsection{Ambipolarity}

Graphene is ambipolar. Ambipolarity means that carriers can be changed continuously between holes and electrons by applying the required gate bias. This is attributed due to the unique band structure of graphene . When negative gate bias is given, the Fermi level drops below the Dirac point due to which a large number of holes are populated into the valance band. And when positive gate bias is applied, the Fermi level rises above the Dirac point, and a large number of electrons are populated into the conduction band.

\subsubsection{Stability}

Graphene nanoribbons are chemically inert even at high temperature .It possess exceptional thermal and chemical stability. Suspended single layer of graphene is stable at very high temperatures even at $2600 \mathrm{~K}$ or above [14] .This exceptional behavior can be attributed to high thermal conductivity of suspended graphene. All these properties make graphene an excellent protective layer.

\subsubsection{Impermeability}

Graphene is highly impermeable, in fact ,it is the most impermeable material found so far. Impermeability of graphene can be attributed to its closely packed structure, high electron-density ,ultrathin structure and high strength. Impermeability of graphene is so high that even smallest element are not able to pass through it and only water vapors can pass through a sheet of graphene. When sea water is passed through the sheet of graphene only water molecules are able move through it and impurities are left behind. Due to its impermeability, Graphene-lined hydrogen fuel tanks can be made. Also, Leak-proof storage of hydrogen is possible with graphene. This makes graphene useful in desalination of water. With $\mathrm{C}-\mathrm{C}$ bond energy $4.9 \mathrm{eV}$ and intrinsic strength $43 \mathrm{~N} / \mathrm{m}$ [15],graphene forms thinnest impermeable membrane.

\subsubsection{Hydrophobicity}

Binding energy per cluster is more than absorption energy of water molecules for graphene. Therefore water forms cluster on graphene sheets. Therefore, graphene is hydrophobic.

\subsubsection{Fractional Quantum Hall Effect}

The quantum Hall effect was the most remarkable phenomena discovered in condensed matter physics in late $20^{\text {th }}$ century. It means that many electrons come together to form new particles which have charge smaller than that of a single electron which is very bizarre. Particles were never seen to behave like this. They join together to form bigger objects or keep their size, but they were never seen to form particles of smaller size. Such particles have exactly $1 / 3,1 / 5$ or $1 / 7^{\text {th }}$ etc. of electronic charge on them. Sometimes, magnetic field get attached to each of these electrons which create other objects. Such composite particles have properties other than the electrons. They do not get deflected by the large value of magnetic field and move along a straight line. The mass of these composite particles is not related to mass of the original electron but originates from interactions of the neighboring particles. Also, associated magnetic field changes characteristics of such composite particles, from half integral particle ,i.e., fermion to integral particle, i.e., boson and back to fermion. And at last some of such composite particles are speculated to fuse together to form pairs. This phenomena is similar to Cooper pair production in superconductivity.

In 1980 an interesting phenomena called integral quantum Hall effect was discovered by Klaus von Klitzing, Michael Pepper, and Gerhardt Dordda [16] .At low temperatures, hall-resistance of a two dimensional electron system is found to have plateaus at the precise values of $\boldsymbol{h} / \boldsymbol{n} \boldsymbol{e}^{2}$, where $\mathrm{n}=$ integer, $\mathrm{h}=\mathrm{Planck}$ 's constant and e =electron charge. When magnetic field is applied the Hall resistance shows the plateaus, the magneto-resistance ,that is, the resistance measured along the direction of the current flow drops to very low 
values. Later, even more intriguing fractional quantum Hall effect was discovered by Horst L. Störmer, Daniel C. Tsui, and Arthur C. Gossard [16]. When temperature of two dimensional electron system is reduced below $2 \mathrm{~K}$, the Hall resistance shows plateaus at the values of $\boldsymbol{h} / \boldsymbol{n} \boldsymbol{e}^{\mathbf{2}}$, where $\mathrm{n}$ is a fraction such as $1 / 3,1 / 7,2 / 3,4 / 5$ and so on [16]. When two dimensional electrons interact with each other in strong magnetic field environment ,Coulomb interaction between the electrons become very strong and a new state of matter is formed due to condensation of these two dimensional electrons. This is called fractional quantum hall effect. The experimental studies confirm anomalous integer quantum Hall effect in graphene. However, graphene has shown only weak signs of fractional quantum hall effect.

Many other intriguing properties of Graphene are being explored by the scientist all over the globe. Recently it has been found that magnetic field of graphene can be switched and off. This further expands the gates for various electronic applications. With so many exceptional properties promises that scientific community is making are many, potential applications of graphene seem to be limitless. The only problem is we still do not know how to use its potential.

\subsection{Applications}

\subsubsection{Medicine}

Large and planar $\mathrm{sp}^{2}$ hybridized, large specific surface area of $2630 \mathrm{~m}^{2} / \mathrm{g}$ [17] and enriched oxygen-containing groups give graphene oxide excellent biocompatibility, solubility and stability. All these properties make graphene oxide multi-functional for various biological and medical applications. Due to its low cost and useful interactions with drug molecules, Graphene Oxide is a being studied for the use in biological and medical applications

\subsubsection{Drug Delivery}

Graphene and Graphene oxide are ideal nano-carriers for effective gene and drug delivery. Graphene oxide used for drug delivery is about one-two nanometer thick, with size staring from a few to 100s of nm [18]. Drug delivery applications of graphene and graphene oxide include delivery of anti-cancer drugs, as a drug carrier for loading pirfenidone in treatment of subarachnoid hemorrhage, poorly soluble drugs, peptides, antibiotics, antibodies, DNA, RNA, genes. Others nano-carriers lack ability to achieve high targeting concentration and efficiency at tumor sites, have limited drug loading capacity, a low degree of functionality, insufficient cell uptake, leads to nonspecific accumulation of the drug in normal cells, which further decreasing the therapeutic efficacy of the drug. Graphene and graphene oxide, on the other hand, are used to prevent unsought drug release into the blood stream during drug transportation and is therefore used for the effective drug transportation of anti-tumor drugs in tumor cells or tissue. They have flexibility and capability to design complex multifunctional drug systems for combined therapies. They have high drug loading capacity. Both covalent and non-covalent modifications can be used to give specific biological activity to G and GO, and also to improve the bio-compatibility and colloidal stability [18].

\subsubsection{Tissue Engineering}

Graphene and Graphene Oxide act as catalyst are in growth, differentiation, and maturation of stem cells. Graphene and graphene oxideare soft memberanes which allow stem cell attachment and growth and increase osteogenic differentiation. Thus they act as a transferable and implantable platform for growth of stem cells .Therefore, G and GO can be thought to act as an alternative material for bone regeneration. Graphenebased materials enhance stem cell differentiation. Human osteoblast-like cells and mesenchymal stromal cells sown on chemical vapor deposition synthesized graphene show higher growth than silicon dioxide [18].

\subsubsection{Devices}

Scientists have made electrodes which can be placed deep into the brain and can connect directly to neurons and transmit their electrical signals away from the body. These electrodes are supposed to help amputees, people with Parkinson's disease, paralyzed people, people with motor control disorder and other neurological disorders.

\subsubsection{Bio-Micro Robotics}

Medical microrobots are being studied for their applications in targeted drug delivery, surgical accuracy, biopsy, faster diagnosis ,hyperthermia, radioactive therapy, scaffolding, surgical ablation in living bodies, stenting, sensing, marking and in hospital housekeeping. Such tasks are preferred to be carried out with micro robots because they are least invasive and precisely targeted. Temperature responsive hydrogelGraphene oxide nano-composite bi-layers closely resemble to the shape of Jellyfish and Venus Flytrap and have physical and chemical properties similar to biological tissues. These polymer networks have unique capability to reversible volume change in presence of external stimuli. They are used in tissue engineering, targeted drug delivery and wound healing. 


\subsubsection{Bio-Imaging}

Bio-imaging is a method that helps us to visualize biological processes with the aim to interfere as little as possible with biological processes. It is used to gain in vivo and in-vitro imaging on the 3-D structure. In also includes methods visualizing biological material that has been fixed for observation. Quantum confinement and edge effects of graphene have shown to induce photoluminescence. Isolated polyaromatic structures and passivated surface defects contribute to the fluorescence of Graphene Quantum Dots [19]. Graphene Quantum Dots are fluorescent carbon-based nano-material that can be used to make effective fluorescent probes for bioimaging due to their chemical inertia and non-toxicity.

\subsection{Electronics \\ 1.3.1 Transistor: \\ 1.3.1.1 FET Transistor}

Like every transistor, a field-effect transistor (FET) is also a three terminal device. These terminals are called source, drain and gate .It is one of the most broadly researched applications of graphene. Its valance band and conduction band meet at Dirac point( a point where electric field and charge carrier concentration are zero). Thus graphene is a zero band-gap semi-metal. 2D Few-Layer graphene shows a strong ambipolar electric field effect, high carrier mobility and large nano-electronic conductance. All these characteristics make it useful for graphene based field effect transistor. Here graphene is used to make the channel. Under the effect of electric field, electrons can be converted into holes and holes into electrons. Under positive gate bias, graphene behave as an electron conductor and under negative gate bias, it behave as a hole conductor.

Because the bandgap is zero, a bilayer graphene transistor has $\mathrm{I}(\mathrm{on}) / \mathrm{I}(\mathrm{off})$ ratio of 100 at 25 degrees Celsius and is about 2000 at 20K [20]. Devices with channels made of large-area graphene cannot be switched off and therefore are not suitable for logic applications. Also, real nanoribbons have rough edges and their widths change along with their lengths. This edge disorder hides the difference in the bandgap between nanoribbons. To get desired bandgap useful for field-effect devices, very narrow nanoribbons with well-defined edges are needed. This is a matter of a great challenge given the processing equipment available at the moment. Nanoribbons with uniform in width and reduced edge roughness can be produced by opening carbon nanotubes. The band structure of graphene can be modified, and it is possible to open a bandgap in three ways: by restricting large-area graphene in 1D to form graphene nanoribbons, by biasing bilayer graphene and by applying strain to graphene. Doping also affects the bandgap in graphene nanoribbons [21]. Both armchair nanoribbons and zigzag nanoribbons are supposed to have a band gap which is inversely proportional to the width of the nanoribbon. But such nanoribbons are not perfect for electronic applications. Band gap for nanoribbons of width below $20 \mathrm{~nm}$ is experimentally shown to be $200 \mathrm{meV}$ [21].

\subsubsection{Single-Electron Transistor}

A single electron transistor is similar to a normal transistor except the channel is replaced by a quantum dot and the dot is separated from source and drain by thin insulators The biggest challenge in utilizing graphene in transistor is that it has no bandgap. But a bandgap can be generated in bilayer graphene using electrostatic gates [22] .Electronic states can be changed drastically by injecting an electron into it. Such small structure is called quantum dot. The electronic states of a quantum dot can be controlled also by controlling the electric potential of the quantum dot. Number of electrons in SET are controlled by the electric potential. The carriers of information in SET are electrons and therefore energy consumed is thought to be very small. Graphene quantum dots can be easily formed by etching because graphene is a single atomic layer thick. But fabrication with precision is still challenging. Single Electron Transistors consist of a sub-micron sized island coupled weakly to source and drain contacts. [22]. When size quantization become important term quantum dot is used instead of single electron transistor.

An electron tunnels in two steps:

source $\rightarrow$ dot $\rightarrow$ drain

Conditions for observing single electron tunneling phenomena (Coulomb blockade) [23]:

1. The Coulomb energy $e^{2} / \mathrm{C}$ should be greater than the thermal energy $\mathrm{k}_{\mathrm{B}} \mathrm{T}$. Otherwise instead of being blocked by the Coulomb energy an extra electron may get onto the dot with thermal energy. A size of the dot should be either small $(<10 \mathrm{~nm}$ at $300 \mathrm{~K})$ or it should be cold $(<1 \mathrm{~K}$ for a $\mu \mathrm{m}$ sized dot).

$$
E_{c}>k_{b} T
$$

2. The residence time $\Delta \mathrm{t}=\mathrm{RC}$ of an electron on the dot needs to be so long that the corresponding energy uncertainty $\Delta \mathrm{E}=h / \Delta \mathrm{t}=h / \mathrm{RC}$ is less than the Coulomb energy $e^{2} / \mathrm{C}$. That leads to a condition for the tunnel resistance between the dot and source/drain:

$$
R>h / e^{2}, \text { where } h / e^{2} \approx 26 \mathrm{k} \Omega
$$


A quantum dot is connected with two electrodes : One electrode is connected to dot through a tunneling junction the other electrode, gate, coupled with quantum dot via insulator. Electrons cannot pass through tunneling. Electrons are injected or ejected to/from the dot.Advantages SETs compared to MOSFETs are low power consumption, good scalability, high output impedance, operation at low temperatures.

\subsubsection{Spin Transport Device}

Electronic devices run due to motion of electric charge in conductors and semiconductors. Spin of electron can also be exploited to create new generation devices called spintronic devices. These devices are expected to be compact, robust and more versatile than conventionally used silicon chips. Traditional spin devices were based on the alignment of the spin relative to external magnetic field or magnetization orientation of ferromagnetic film [24]. New approach to such devices is that spin degree of freedom can be added to traditional charge based semiconducting devices or spin alone can be exploited to create spintronic devices. These devices are based on the fact that spin of electron can be controlled by an external magnetic field and polarize the electrons, such electrons are used to control the electric current. Spin based devices such as magnetic memory, spin based FET, spin based LED, encoders, decoders, modulators, magnetic recording etc are a few of emerging applications of spintronics . [25]. Graphene is thought to be a promising material for spintronics because it has low intrinsic spin-orbit interaction and low hyperfine interaction of the electron spins [26]. Information can be carried out in the form of the flow of electron-spin polarity. Such devices are much more useful than conventional devices that use electric current. Graphene is an ideal candidate for such devices because electrons are least likely to be perturbed by the carbon nuclei which is due to the fact that carbon atoms are light. Also, spin injection into graphene is possible and electrons diffusion takes place in graphene keeping the spin polarity.

\subsubsection{Conductive Ink}

Inks are used to color the surface. Conductive inks function as a conductor. Conductive inks are used in many applications such as sensors, antennae, touch screens, printed heaters and other electronic devices. High quality repeatable and reliable patterns can be made on paper or plastic substrates using SGO inks or FGO inks by simple inkjet printer. The patterns so formed are of controllable thickness and line width and they retain their high conductivity. Conventional inks in inkjet inks contain particles of size in the range of 100-400 nm [27]. If graphene is taken as the base conducting filler the size of graphene materials in this range is the key to obtain images of improved quality. The inks can be stored for several months without any aggregation. Viscosity and surface wet-ability of substrates are other important factors which affect the printing process and image quality. Although patterns when printed on PET or PI were not continuous. The conductivity of the patterns was observed to increase when the number of times the patterns printed increased. FGO ink has higher electrical conductivity than GO ink. [28]

\subsubsection{Frequency Multiplier}

Frequency multiplication is an essential part of radio communication and broadcasting. Frequency multiplier , as the name suggests, gives the output signal frequency integral multiple of input signal frequency. Unique ambipolar transport property of graphene and high mobility of its charge carriers make graphene useful for its application as frequency multiplier, that can operate up to $1.4 \mathrm{GHz}$ with high purity in output signal [29]. A high efficiency frequency multiplier can be made by using top gated G-FET in which substrate used must be intrinsic silicon.Frequency doubling using G-FETs has conversion efficiency greater than $90 \%$.

\subsubsection{Quantum Dots}

Graphene Quantum dots have many applications such as photovoltaic cell, organic LEDs, fuel cells, bioimaging and biosensing devices, environmental monitoring devices and many others. From its applications in energy harvesting, storage to bioanalytics, GQDs is one of its kind. Potential applications include batteries, supercapacitors, field emitters, and interconnects, nano electromechemical systems (NEMS), labs-on-chips, or nanometer-scale switches. Now, what actually is a quantum dot. Electronic states can be changed drastically by injecting an electron into it. Such small structure is called quantum dot. The electronic states of a quantum dot can be controlled also by controlling the electric potential of the quantum dot. Graphene quantum dots can be easily formed by etching because graphene is a single atomic layer. The major challenge here is production of high-quality graphene quantum dots. Present day production methods do not result in well-defined particles or are not efficient. Commercial applications of Graphene Quantum Dots will rapidly evolve only if GQDs of good quality are available on a large scale. GQDs is being thought as future of carbon materials and of environmentalfriendly devices. GQDs are more efficient and less toxic alternative of currently used quantum dots. 


\subsubsection{Hall Effect Sensors}

When a current carrying conductor is placed in a magnetic field, a potential difference at the ends of the conductor is developed which is proportional to the current and magnetic field. This is called Hall effect. It is the basic principle for many magnetic field measuring instruments. A hall effect sensor generates an electrical signal. Such kind of sensors usually contain a magnetic system, which converts the physical quantities such as position, speed, temperature, current etc into the magnetic field which in turn can be sensed by Hall-effect sensors. Magnetic field sensors are required in many applications vehicle production, gaming consoles, electronics industry, security, healthcare, aerospace, defense industry, etc. Such sensors demand high accuracy in measurement of position, proximity, and motion. The present graphene-based Hall sensor has current sensitivity as high as 5700 where silicon devices have a current sensitivity of mere 100 [30]. Voltage sensitivity is also 28 times higher $\mathrm{Sv}$ Si-based sensor is 0.1 and for Graphene $\mathrm{hBN}$ sensors it is 2.8. [30]

Table 1: Comparison of figures of merits of Hall sensors at room temperature [30]

\begin{tabular}{|c|c|c|c|c|}
\hline Material & $S_{1}$ & Sv & $B \min \bullet W$ & \\
\hline & [V/AT] & VT] & & \\
\hline $\mathrm{Si}$ & 100 & 0.1 & 1500 & NA \\
\hline GaAs & 1100 & NA & 8000 & NA \\
\hline InAsSb & 2750 & NA & 50 & NA \\
\hline Graphene & 2093 & 0.35 & 5000 & Air \\
\hline Gr-hBN (SI) & 4100 & 2.6 & 150 & Vac \\
\hline Gr-hBN (SI) & 4000 & 3 & NA & Air \\
\hline Gr-hBN (S2) & 5700 & 2.8 & NA & Air \\
\hline
\end{tabular}

Thus graphene especially graphene encapsulated in hexagonal boron nitride is a promising material in making Hall sensors.

\subsubsection{Transparent Conducting Electrodes}

Transparent Conducting Electrodes (TCEs) are widely used in optoelectronic devices like solar cells, LEDs, liquid crystal display, and display screens. There is continuous research being conducted to increase the performance. Currently both high temperature and low temperature manufacturing processes are being used to produce TCEs. Indium tin oxide (ITO) and fluorine tin oxide (FTO) are being used as window electrodes in optoelectronic devices. The problem with these materials is that they are brittle, their quantity limited, they are quite costly, their chemical stability is also very low due to which they may diffuse into the active organic-LED layers and corrode the device. Therefore, present transparent conducting films have serious drawbacks. Graphene has a work function of $4.5 \mathrm{eV}$ to $4.9 \mathrm{eV}$ [31] which is similar to Indium tin oxide. It is also flexible, cheap and does not diffuse into the device. This makes graphene ideal candidate for transparent conducting films (TCFs). Graphene based transparent electrodes are used in three types of photovoltaic devices: organic, inorganic-organic hybrid, and dye-sensitized solar cells. Large area, continuous, transparent, and highly conducting few-layer graphene films deposited by the chemical vapor deposition (CVD) process show highest power conversion efficiency (PCE, $\eta$ ) of $1.71 \%$ in the organic photovoltaic (OPV) solar cells [32]. Graphene films are modified with pyrene butanoic acid succidymidyl ester which is responsible for such high value of power conversion efficiency [32]. This performance is $55.2 \%$ of the power conversion efficiency of a control device based on indium tin oxide ITO [33]. CVD graphene is transferred onto a flexible PET substrate for fabrication of OPV devices. Graphene/PET-based and ITO/PET based OPV devices show performances with of 1.18 and $1.27 \%$, respectively [32]. Importantly, the CVD grown graphene-based solar cells show the outstanding capability to be operated under bending conditions with bending angles up to $138^{\circ}$, while the ITObased devices display cracks and irreversible failure under bending of only $60^{\circ}$ [32]. Therefore, the epitaxial grown graphene is highly flexible, transparent and continuous and electrodes made out of it have potential to replace ITO in flexible photovoltaic applications. In place of CVD-grown graphene reduced graphene oxide film can also be used as a transparent electrode. 


\subsubsection{Light-emitting devices}

Organic light-emitting diodes are based on electroluminescence. These diodes contain two layers layer at least one of which is transparent. Due to diffusion, these carriers move and recombine to form excitons. When these excitons come to the ground state, a photon is emitted. OLEDs are widely used because of their high image quality,flexibility, low power consumption, and ultrathin and light device structure. Sleek displays are made up of OLEDs, such as ultrathin televisions, computer monitors, camera screen and mobile phones. Currently, Indium tin oxide (ITO) is being used as a conductive film but ITO is brittle, the quantity of Indium available is limited that is why it is quite costly, chemical stability of Indium is also low due to which it may diffuse into the active OLED layers and corrode the device. Thus, transparent conducting films have serious drawbacks. Graphene has a work function of $4.5 \mathrm{eV}-4.9 \mathrm{eV}$ [31] which is similar to Indium tin oxide. It is also flexible, cheap and does not diffuse into the device. This makes graphene ideal candidate for transparent conducting films(TCFs).

\subsubsection{Photodetectors}

Photodetectors contain $p n$ junction diode that convert light energy into electrical energy which can be measured by the photodetector. Photon absorbed at the junction results in charge carriers to be excited, the excited carriers jump from the VB (valence band) to the CB (conduction band), which result in electric current. Many devices such as remote controls, smoke detector, televisions and DVD players are based on photodetection. Photodetectors based on goup-IV and p- or n- semiconductors become transparent when the incident energy is smaller than the bandgap, therefore have a wavelength limit( [34] .Graphene absorbs light from the ultraviolet to terahertz range [35]. As a result, wavelength range over which graphene-based photodetectors can work is quite broad. The response time is governed by the carrier mobility and the mobility of Graphene is quite high which results in faster functioning. The photo thermoelectric effect, in which light energy is first converted into heat energy and then into electric energy [34], can play an important role in photocurrent generation. Thus photo thermoelectric graphene photo-detectors may be possible.

\subsubsection{Touch screens}

Touch screens are sensitive displays that on touching allow human interaction with the computer. It detects presence and location of the touch within the. They are used in many applications such as cell phones, computer, digital displays and digital cameras. They allow quick and exact interaction of the user with the content of the device. There are two types of touch screens that are being used: resistive and capacitive touch screens. A resistive touch panel consists of a conductive substrate, a liquid-crystal device front panel, and a transparent conducting film. When the screen is touched the front-panel film comes in contact with the transparent conducting film. The location of the contact point can be calculated on the basis of the resistance values. Capacitive touch screens on the other hand cause change in the electric field of the screen. The human body is conductive, this conductivity is used as input in the capacitive screen. A change in the electric field occurs when the touch screen is touched which is registered and located by the processor. That is why it is difficult to operate the capacitive touch screen when wearing the gloves. Currently, Indium tin oxide (ITO) is being used as a conductive film but ITO is brittle, the quantity of Indium available is limited that is why it is quite costly, chemical stability of Indium is also low due to which it may diffuse into the active OLED layers and corrode the device. Thus, transparent conducting films have serious drawbacks. Graphene has a work function of $4.5 \mathrm{eV}$ [31] which is similar to Indium tin oxide. It is also flexible, cheap and does not diffuse into the device. This makes graphene ideal candidate for transparent conducting films(TCFs).

\subsubsection{Optical limiters}

Optical limiters block high intensity radiation but allow low intensity radiations to pass through them. They are used to protect optical sensors, human eye and other electronic devices from high intensity laser beam. They have a high transmittance for low incident light intensity beams and low transmittance for high-intensity beams. They contain nonlinear materials that remain transparent at low intensity of light bit become opaque when intensity of light cross a certain limit. Present day optical limiters have non-linear optical material have issues of overheating when exposed to high level radiation. High level of radiation may even cause an irreversible damage to the device. They are simple, compact and cheap. Till now, no passive optical limiter can work over the entire visible and near-infrared range. Semiconductors, organic molecules, liquid crystals and carbon-based materials, Fullerenes and their derivatives, Carbon NanoTube dispersions, all these materials have good optical limiting performance, in particular for nanosecond pulses at 532nm and 1,064 $\mathrm{nm} \mathrm{[36].} \mathrm{In}$ graphene-based optical limiters, the incident light energy is converted into heat energy which creates bubbles and micro-plasmas which result in reduced transmission. 


\subsubsection{Terahertz devices}

Graphene devices can be used for scanning through objects (like dust, walls, tissues, paper, cardboard, textiles, plastic, wood, and ceramics etc.), Body Scanner, Drugs and ERC's inspection, Tumor diagnostic, bomb detection, check for defects and also quick wireless data transfer. Electronic and optical properties of single layer graphene and few layer graphene can be adjusted with the help of electric or magnetic fields or an optical pump. Also, graphene has high carrier mobility at room temperature, linear gapless spectrum, possibility to change its dielectric constant, frequency independent visible optical absorption. This makes graphene a suitable material for modulator, sources and detector working across the entire far-infrared range. Biomedical imaging, security, remote sensing, and spectroscopy require radiation in the range of $300 \mathrm{GHz}-10 \mathrm{THz}$ [37]. Earlier devices had costly and inefficient sources and detectors.

\section{Conclusion}

Graphene, Graphyne, Borophene, Germanene, Silicene, $\mathrm{Si}_{2} \mathrm{BN}$, Stanene, Phosphorene, Molybdenite, two-dimensional metal layers etc. are various two-dimensional materials discovered in recent years and graphene was first to be discovered. Graphene is thought to be perfect two-dimensional crystal due to intriguing properties. It is versatile two dimensional atomic thick carbon lattice. Versatility of graphene lies in its properties from physical to electronic. Due to these properties graphene can be used in many applications. Biomedical applications such as drug delivery, cancer treatment, bio-imaging, micro-robotics; electronics applications such as touch screens, transistors, integrated circuits, solar cells, LEDs, spin transport devices, conductive ink, frequency multiplier, Hall effect sensor, quantum dots, frequency multiplier, terahertz devices, photo- detectors, optical limiters; Energy generation ,storage , fuel cells, super capacitors, batteries ;optical Modulator, ultraviolet lens, infra red light detection ,photo detector, pressure sensor, body sensor, contaminant removal, water Filtration, nano-antennas, radiowave absorption, lubricant ,waterproof coating, ethanol distillation etc. are few applications which has caught attention of science community. It also has potential to replace present day benchmark materials like silicon in transistor, steel as a building material (graphene is 100 times stronger than steel). But the question is, are properties of graphene can actually be used in present day applications.

\section{Bibliography}

[1] A. K. Geim, "Random Walk to Graphene (Nobel Lecture)," Angewandte Chemie International Edition, 2011

[2] S. W. S. N. H. S. S. \&. B. M. Balendhran, "Elemental analogues of graphene: silicene, germanene, stanene, and phosphorene," vol. 11, no. 6, pp. 640-652, 2015 .

[3] H. P. C. A. F. G. O. \&. H. U. Boehm, "Das adsorptionsverhalten sehr dünner kohlenstoff-folien," Zeitschrift für anorganische und allgemeine Chemie, pp. 119-127, 1962

[4] L. R. S. \&. C. J. Foa Torres, "Introduction to Graphene-Based nanomaterials: from electronic structure to quantum transport," Cambridge: Cambridge University Press, pp. 1-10, 2014.

[5] A. D. S. A. J. S. J. G. S. a. C. T. G. Cameron J Shearer, "Accurate thickness measurement of graphene," Nanotechnology, pp. Volume 27, Number 12, 2016

[6] C. W. X. K. J. W. \&. H. J. Lee, " Measurement of the elastic properties and intrinsic strength of monolayer graphene.," science, pp 385-388., 2008.

[7] T. M. Radadiya, " A PROPERTIES OF GRAPHENE," European Journal of Material Sciences, pp. 6-18, (2015).

[8] J. P. Lu, " Elastic properties of carbon nanotubes and nanoropes," Physical Review Letters, p. 1297., (1997).

[9] K. I. K. J. S. Z. J. M. K. G. F. J. H. P. K. a. H. L. S. Bolotin, " Ultrahigh electron mobility in suspended graphene," Solid State Communications, pp. 351-355, (2008).

[10] J. B. A. \&. B. A. Moser, "Current-induced cleaning of graphene," Applied Physics Letters, pp. 91, 163-513 , 2007.

[11] V. V. a. A. K. R. Eric Pop, "Thermal properties of graphene: Fundamentals and applications," MRS Bulletin, pp. vol. 37, pp. 12731281, (2012).

[12] I. R. A. Shahnawaz Uddin, "GRAPHENE: A FASCINATING MATERIAL," ICRISTME, pp. 89-97, 2017.

[13] A. K. D. S. Sahoo, "GRAPHENE: A NEW STAR IN MATERIAL SCIENCE," Department of Physics, National Institute of Technology, p. http://physics.unipune.ernet.in/ phyed/27.3/1374.pdf.

[14] W. R. B. G. B. A. B. M. K. Kwanpyo Kim, "High-temperature stability of suspended single-layer graphene," physica status solidi, pp. 11, 302-304, 2010.

[15] V. Berry, "Impermeability of graphene and its applications," Carbon, pp. 1-10, 2013.

[16] "The Quantum Hall Effect," http://www.phys.columbia.edu/ w3081/exp_files/qhenotes.pdf.

[17] K. S. S. a. C. N. R. R. H.S.S. Ramakrishna Matte, "Synthetic Aspects and Selected properties of graphene," Intech, pp. 1-11, 2011.

[18] ～L. z. M. L. Z. He Shen, "Biomedical Applications of Graphene," Theranostics, pp. 283-294, 2007.

[19] L. L. W. ,. a. X. Q. .. ". N. Feng, "New horizons for diagnostics and therapeutic applications of graphene and graphene oide," advanced materials, 2013.

[20] D. B. F. Y.-m. L. a. P. A. Fengnian Xia, "Graphene Field-Effect Transistors with High on/off current ratio and large transport gap," Nano letters, pp. 715-718, 2010.

[21] A. S. a. P. M. A. Deep Jariwalaa, "Graphene synthesis and band gap opening," Department of Metallurgical Engineering, Institute of Technology, 2011.

[22] J. G. F. M. S. S. E. S. A. J. S. H. T. F. S. D. C. S. T. Ihn, "Graphene single electron Transistors," Materials Today, pp. 44-50, 2010.

[23] J. G. F. M. S. S. E. S. A. J. S. H. T. F. S. D. C. S. b. K. E. T. Ihna, "Graphene single-electron transistors," Materials Today, pp. Volume 13, Issue 3, Pages 44-50, March 2010.

[24] D. D. A. R. A. B. M. D. v. M. L. R. A. Y. C. D. M. T. S. A. Wolf, "Spintronics: A Spin-Based Electronics Vision for the Future," sciencemag.org, pp. 1488-1494, may,2012. 
[25] K. R. U. W. a. C. R. R. Raju, "Honeywell Technology Solutions, Bangalore JNCASR,," Stockholm University, 2008.

[26] C. J. M. P. H. T. J. \&. B. J. v. W. Nikolaos Tombros, "Electronic spin transport and spin precession in single graphene layers at room temperature," Nature, pp. 571-575, 2007.

[27] S. M. a. M. B. Moshe, "Patterning of Organic Nanoparticles by Ink-jet Printing of Microemulsions," Langmuir, pp. 19 (3), pp 939942, 2002.

[28] Y. H. ,. J. L. X. W. a. Y. C. Lu Huang, "Graphene-Based Conducting Inks for Direct Inkjet Printing of Flexible Conductive Patterns and Their Applications in Electric Circuits and Chemical Sensors," Tsinghua University Press and Springer-Verlag Berlin, p. 4(7): 675-684, 2011.

[29] K. N. P. F. C. W. M. H. C. P. M. R. S. R. S. K. B. D. A. Michael E. Ramon, "Three-Gigahertz Graphene Frequency Doubler on Quartz Operating Beyond the Transit Frequency," IEEE TRANSACTIONS ON NANOTECHNOLOGY, pp. VOL. 11, NO. 5., 877883,2012

[30] A. A. S. M. O. W. T. D. N. C. S. Jan Dauber, "Ultra-sensitive Hall sensors based on graphene encapsulated in hexagonal boron nitride," arxiv.org, 2015.

[31] J. H. H. J. S. S. T. S. K. C. C. H. J. W. a. J. Y. P. Jong-Hun Kim, "Work function engineering of single layer graphene by irradiation-induced defects," AIP Publishing, pp. 103, 171604-1 , 2013.

[32] Z. Y., ,. S. W. ,. X. Q. ,. Q. H. ,. Q. Z. ,. Y. ,. F. B. ,. a. H. Z. Xiao Huang, " Graphene-Based Materials: Synthesis, Characterization, properties and application," wileyonlinelibrary.com, pp. 1876-1902, 2011

[33] X. C. Y. Z. F. Z. a. K. P. L. Yu Wang, "Large area, continuous, few-layered graphene as anodes in organic photovoltaic devices," APPLIED PHYSICS LETTERS , 2009.

[34] B. E. A. \&. T. Saleh, "M.C fundamentals of photonics," Wiley, pp. ch 18,784-803, 2007.

[35] R. R. e. a. Nair, "Fine structure constant defines transparency of graphene," Science 320, , p. 1308-1308, (2008).

[36] A. A. Ryzhov, "Optical Limiting Performance of a GaAs/AlAs Heterostructure Microcavity in the Near-Infrared," arxiv.org, 2017.

[37] A. K. Subbiah Alwarappan, "Graphene-Based Materials: Science and Technology," CRC Press, 2017.

[38] H. L. Stormer, "The fractional quantum hall effect," Department of physics and applied physics, Columbia university, 1998

[39] A. K. S. K. S. S. a. A. G. C. N. R. Rao, "Graphene: The New Two-Dimensional Nanomaterial," angewandte chemie, pp. 7752-7777, 2009 .

[40] N. Tombros, "Electron spin transport in graphena and carbon nanotubes," Zernike Institute for advanced physics, 2008

[41] M. J. a. R. H. Silsbee, "Coupling of electronic charge and spin at a ferromagnetic-paramagnetic metal interface," PHYSICAL REVIEW B, 1988

[42] B. H. X. M. Z. Z. Z. Z. Z. G. P. a. H. C. Chuantong Cheng, "Frequency conversion with nonlinear graphene photodetector," Nanoscale, royal society of chemistry, pp. $001-8,2016$ 